\title{
Research on Value Based Heuristics Miner for Product Service System
}

\author{
Xuequan Zhou ${ }^{1,2}$ \\ 1. Research Center of Intelligent \\ Computing for Enterprises \& Services \\ Harbin Institute of Technology \\ Harbin, China \\ zhouxq@hit.edu.cn \\ Yuqi Liu \\ Research Center of Intelligent \\ Computing for Enterprises \& Services \\ Harbin Institute of Technology \\ Harbin, China \\ 180300415@stu.hit.edu.cn
}

\author{
Yi Tan \\ Research Center of Intelligent \\ Computing for Enterprises \& Services \\ Harbin Institute of Technology \\ Harbin, China \\ isTanyi@163.com \\ Kexin Tan \\ Research Center of Intelligent \\ Computing for Enterprises \& Services \\ Harbin Institute of Technology \\ Harbin, China \\ 13271392464@163.com
}

\author{
Gregory Zacharewicz \\ Laboratoire des Sciences des Risques \\ (LSR) \\ IMT Mines Ales \\ Alès CEDEX, France \\ gregory.zacharewicz@mines-ales.fr \\ David Chen \\ 2. IMS UMR CNRS 5218 \\ University of Bordeaux \\ Talence CEDEX, France \\ david.chen@u-bordeaux.fr
}

\begin{abstract}
Manufacturing enterprises integrate products and services, to improve and expand business processes from the perspective of service, so that they can realize the management of the whole life cycle of the product and obtain more benefits. The original process mining algorithm cannot support the establishment of customer-centered service process model, nor can it describe the value creation mechanism of service stakeholders in the service process. To solve this problem, this paper takes the manufacturing product service system as the background, improves the flexible heuristic miner, which can mine customer-oriented service process model from the event $\log$, and extracts the actors and value information of service activities, so as to build the service-oriented process value model. We verify the improved heuristic miner through a case of air conditioner product service system.
\end{abstract}

Keywords—product service system, service value, improved heuristic miner, process mining

\section{INTRODUCTION}

At the present time, the homogeneity of products is significant, and the market competition is fierce. To obtain the growth of value, the integration chain of products and services is becoming more and more complex, the co-creation of service value is gradually getting people's attention, and the source of value is increasingly transferring to customer demand [1]. Enterprise business is classically productoriented, but service approach is a different concept. It is more concerned about how to realize customer expectation value. With customer demand as the core, the service provider cooperates with customers to create value in the whole service process. Product service system is a system composed of products, services, participant network and supporting infrastructure to continuously meet customer needs [2]. In the manufacturing industry, PSS usually provides customers with products integrated into services, and products serve as the carrier of services. At the same time, services also bring heterogeneity to the providers and enhance the competitive advantage of them. PSS connects enterprises, customers, and the environment together through value creation, which can increase the value-added of services, bring personalized services with heterogeneity, and improve profit margins.

Process mining is techniques to obtain process knowledge and extract structured Process models from the collection records of the actual execution of an enterprise. It can discover, monitor, and improve the actual system behavior [3]. The $\alpha$ algorithm is a milestone algorithm for process mining. It constructs a process model represented by Petri net by finding out the activities of the process and mining the four basic relationships in the log, namely, following, concurrent, causal, and irrelevant. But due to the $\alpha$ algorithm cannot solve the problem of noise and identify anomalies, researchers propose a series of new algorithms from different angles to solve these problems, such as inductive mining algorithm, heuristic mining algorithm and so on.

However, the traditional product-oriented system mainly focuses on the manufacturing and use value of physical products. It considers the integration and optimization of different manufacturing resources and pays little attention to the services and the integration of products and services, which are obviously different from products. Under the trend of servitization, the original business process mining algorithms cannot meet the needs of service scenarios and cannot mine the value information in the service process, so it is necessary to optimize these algorithms from the perspective of service and value creation. Based on the domain knowledge and characteristics of manufacturing product service system, this paper studies and improves the flexible heuristic miner. The improved algorithm can extract the service stakeholders and their related value information from the event log and establish the service process value model. The content of the model is richer, and the application scenarios are wider, which is more suitable for the current needs of manufacturing enterprises from product manufacturing to product service transformation.

\section{RELATED WORK}

This section introduces the related research work and theoretical basis, including product service system and heuristic miner.

\section{A. Product Service System}

The "servitization" was first introduced by Vandermerwe and Rada [4], which refers to an integrated product-service package offered by manufacturing enterprises in addition to physical products. The integration package is gradually dominated by services, and services become the main source of value creation. With the deepening of the research, servitization research develops in many directions, which can be divided into input servitization 
and output servitization. In the direction of output service, product system is gradually changing to product service system. Product service system, initially considered as a competitive system composed of products, services, participant network and supporting infrastructure, can continuously meet customer needs and has less environmental impact than traditional business model [5]. PSS has the following characteristics: it has a process of transforming from traditional design and sales of physical products to an ecosystem of organic integration of tangible products and intangible services [6-7]; it is customer-oriented, with the goal of delivering services to achieve customers' requirements, and the products are the carriers of services; it seeks a balance between the exchange value at the manufacturing end and the use value at the consumption end, reconciliates the conflict between the two, realizes the synergy of interests through the integration and complementarity of resources, and realizes the win-win of value increment; it is under the service-oriented logic, the key creator of value is the customer, and the core of value is the realization of use value, rather than exchange value [8]; and the value is created by resource integration and application between service providers and customers.

According to the degree of servitization, the PSS can be classified into three types [4]: product oriented, mainly focusing on product design, manufacturing and sales, providing some supplementary services; use-oriented, focusing on product related services or service-oriented products, providing the right to use the product rather than ownership; result-oriented, in which the product is a part of the service, delivering value to customers through a customized portfolio of products and services and with attention to the satisfaction of customer needs.

\section{B. Heuristics Miner}

Process mining has been emerging for two decades ago and many different algorithms has been developed, such as the algorithm based on the ADONIS model, the algorithm mining models of concurrent workflows], the algorithms based on computational intelligence, and those related to big data, , etc. Depending on their functions, the algorithms can be classified into algorithms of model creation, information extraction, and model improvement [9].

In 2006, Weijters and Van der Aalst proposed an algorithm called Heuristic Miner (HM) [10], which focuses on the control flow of the process model. The algorithm can process the data containing noise well and extract the correct process model from it. The sequences of events in a case are derived from the timestamp in the event logs and then calculating related frequency-based metrics to determine the dependencies between activities. Next, constructing the dependency graph with reliable connections. Three threshold parameters are utilized for the acceptance of dependency relations: dependency threshold, positive observation threshold, and relative-to-best threshold. Then the short loops of length 1 and 2 are captured respectively by the calculation of their dependency measurements. All length 1 loops should be determined first for avoiding mistakes of identification. After that, the choice and parallel relationships are derived from the dependency graph and the event log that has the sequence of tasks. And with computing the inputs and outputs of each task, the basic process structure is obtained. Finally, identifying the long-distance dependencies which indicate cases with the indirect relationship of two activities. Up to here, the resulting process model is established. To avoiding explicit modeling of invisible activities, the process models are represented as causal nets in the algorithm. Six years later, the Flexible Heuristics Miner (FHM) was proposed, which is the updated version of HM. The FHM extends the dependency graph of the original HM algorithm, trying to find a representation with good performance in practice while easily understandable [11]. Presenting in the new process representation language called augmented C-net, the resulting model from FHM is far more comprehensible, especially in the cases of nontrivial structures, low-structured domains, and noise. In the augmented C-net, using bags replacing sets of the C-net, so it's possible to indicate the number of the split/join patterns that appear. These frequencies are useful for computing valid patterns of splits and joins.

\section{IMPROVEd HeURISTICS MineR FOR PRODUCt SERVICE SYSTEM}

Different from the existing process mining algorithms, the improved heuristic miner for PSS in this paper mines the customer-centric service process value model. In order to further explain the difference between the two and explain the content of the algorithm, this section will first describe some relevant concepts, and then elaborate the algorithm ideas and steps in detail.

\section{A. Service and Value Creation}

According to reference [20], a service process is "an ordered set of activities that are accomplished collaboratively by certain people, organizations, or automated resources in order to serve the customer's needs and create value". It describes the process by which the service provider offers services responding to customer needs. In the PSS context, the products and services are merging and increasingly evolving into a whole. Therefore, the service process here is not distinguished by checking the existence of the product manufacturing or service offering, but by identifying whether the process is customer-oriented or not. The service process is made up of different activities, and each activity is done by one or any number of cooperative actors. Throughout the activity, the actors participate in the value creation and exchange, meanwhile, their own value expectations are satisfied. Service process emphasizes the idea of customeroriented, which takes customer demand as the starting point of the process, and the delivery of service to customers as the end of the service. In order to use process mining techniques to identify service process and service value from event logs, the following formal definition of service process model is made by referring to Reference [12].

Definition 1 The service process model is a service process C-net, denoted as $S P M=\left(T, C, I, O, t_{s}, t_{e}\right)$, where,

- $\mathrm{T}$ is a finite set of all activities;

- $\mathrm{C} \in \mathrm{T} \times \mathrm{T}$ is the finite set of causal relations;

- $I: T \rightarrow I(T)$ is the mapping function of inputs, where $t \in T, I(t)=\left\{t^{\prime} \mid\left(t^{\prime}, t\right) \in C\right\} ; O: T \rightarrow O(T)$ is the mapping function of outputs, where $t \in T, O(t)=\left\{t^{\prime} \mid\left(t, t^{\prime}\right) \in C\right\}$;

- $t_{s} \in T, t_{s}$ is the first activity in the service process, triggered by the customer.

- $t_{e} \in T, t_{e}$ is the activity where the service is delivered, and the customer must be actor of the activity. 
The definition of the service process emphasizes the involvement of the customer in the initial activity of the process and in the activity of service delivery.

Definition 2 A service process value model refers to a service process $\mathrm{C}$-net annotated with value information, denoted as $S P V M=\left(T, C, I, O, t_{s}, t_{e}, A, V\right)$, where,

- $T, C, I, O, t_{s}, t_{e}$ are the same in the service process model $S P M$;

- $A$ is the set of actors of activities, $\forall a \in A, \exists t \in T$, so that $a$ is the actor of $t$;

- $\quad V$ is the set of values of activities, and $V(A \times T) \cup(T$ $\times A)$ is the value inputs and outputs of activities and actors.

\section{B. Idea of Improving the Heuristic Miner for PSS}

The improved algorithm proposed in this paper is used to mine the service process model of PSS and determine the stakeholders of the service and the value they create in the service activities. The stakeholders include service providers, customers, and partners of service providers. Based on the above definition of service process model and service process value model, the improvement idea of heuristic miner for PSS is shown in Figure 1.

Firstly, the original event log that stores the service process running records in the enterprise information system is filtered. Filtering operation includes filtering noise, incomplete process, and non-service process. By judging whether the first activity of the process is triggered by the customer and whether there is service delivery in the process, the integrity of the service process is determined. If one item is not true, the process is not a complete service process instance. After filtering, a valid service event log is obtained. Based on the valid event log, the operation of mining causal matrix and extracting actors and values information can be performed concurrently. Mining the causality matrix is to identify the connection between activities and get both the sequence and all direct-follow connection of activities. And extracting the actors and values information refers to identifying relevant actor attributes and value attributes through the analysis of activity attributes in log. After the causality matrix is obtained, the dependency relationship between two activities can be further judged by calculating the four basic relations and the correlation index of short cycle relations, and then the activity dependency graph can be mined. On the other hand, the causality matrix and actor information are combined to find out the relationship between activities and actors and construct the activity-actor map. Combined with domain knowledge and based on actors and values information, the mapping relationship between actors and values is analyzed, and the actor-value map is formed. Finally, starting from the dependency graph, according to the measurement formula of AND / XOR-split / join relationship, the selection and parallel relationship among multiple activities are calculated and determined, and then the nonlocal dependency relationship is determined by using the calculation index of long-distance dependency relationship, and the service process value model is constructed by combining the actor-value map.

\section{1) Actors and Values in the Service}

In the manufacturing industry, service providers are usually required to cooperate with their partners to provide products and services to meet the complex needs of customers. Hereby, according to the roles of these participants in the service, they can be divided into three types: customer, service provider and its partners. The customer refers to the consumer individuals or organizations in the service system, the proposer of the service demand, and the ultimate realization of the value. The service provider is the provider of product services in the service system, and it is the core enterprise to complete the service process and meet customer service needs. The partners are the participating enterprises or individuals that assist service providers to complete the service together, including suppliers, logistics service providers, maintenance service providers, etc.

In the product service system, the activities in the service process are the carriers of value, and all the actors involved in the completion of the activities, that is, the stakeholders, should create value. Service value is the goal of actors in the service. In general, actors always expect maximum output value with minimum input value. Considering the dimension and granularity of service value and the perception of participants, we can extract value information from event logs

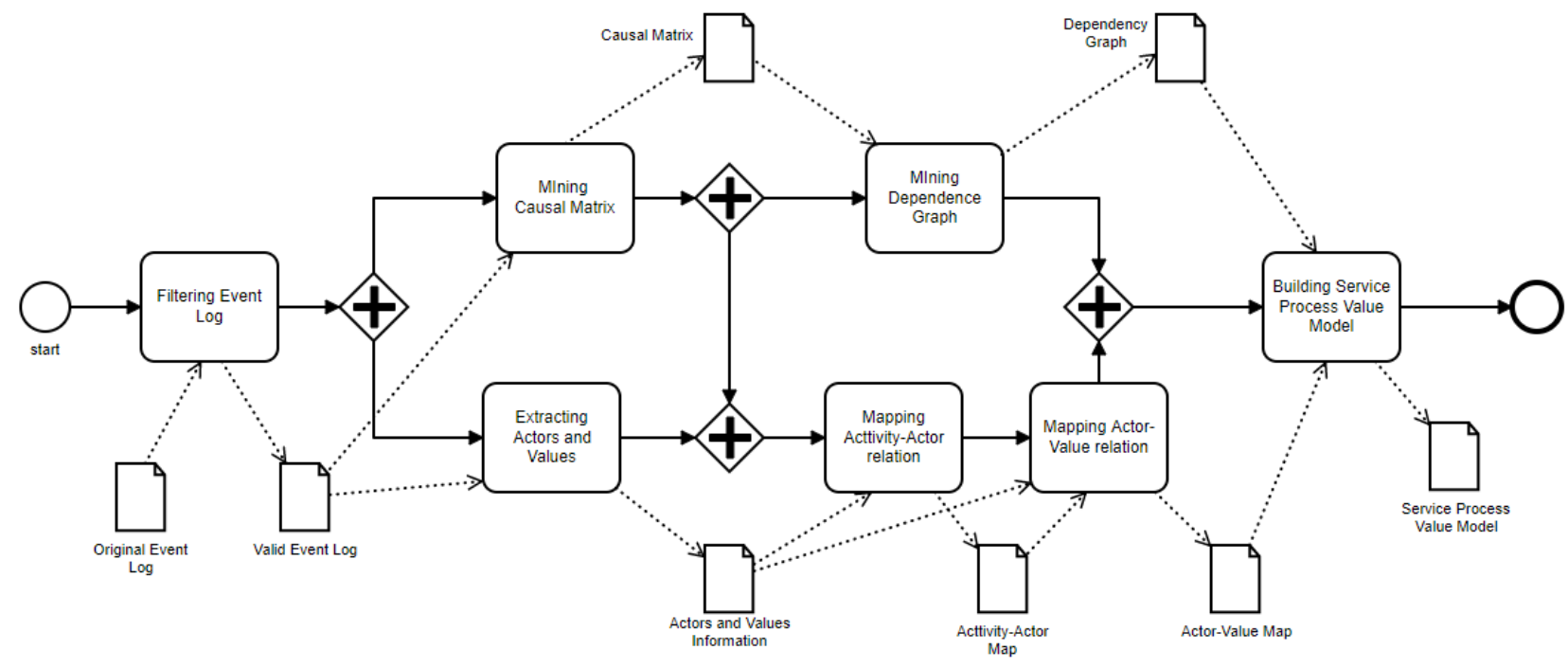

Figure 1. Idea of Improving the Heuristic Miner for PSS 
from three aspects: general value, domain-related value and actor-related value. General value refers to the common characteristics of the value created by the actors in the product service system, which is not affected by the product and service types. These values mainly include time, space (state), delivery, cost, price, etc. Domain-related value refers to the characteristics that the value created by the entity in the product service system is related to the domain of the product and service. The value of product service system is different in different fields. Actor-related value refers to the characteristics of the value created by the actor in the product service system that are related to itself. In the process of service, all actors will consider the actual situation and their own value expectations, and the relevant factors will have an impact on the value of service. We can map these extracted service value to three dimensions of value, namely time and space, profit and experience [12]. Since the event log is a collection of service process execution records, and each activity in the model corresponds to many specific activity instances, after extracting the value of all specific activities, we need to abstract them. Then we must get the average value or value range. For example, the time value of an activity is often the average time spent by the participants on the task.

\section{2) Activity-actor Map}

For each activity in the service process, there are corresponding stakeholders involved in completing the activity and generate value-added. The value-added of an activity is closely connected with the actors. The value created by the same actor in different activities is different, and the value-added of different actors participating in the same activity is also different. Therefore, it is necessary to distinguish the different actors of the activity in the service process.

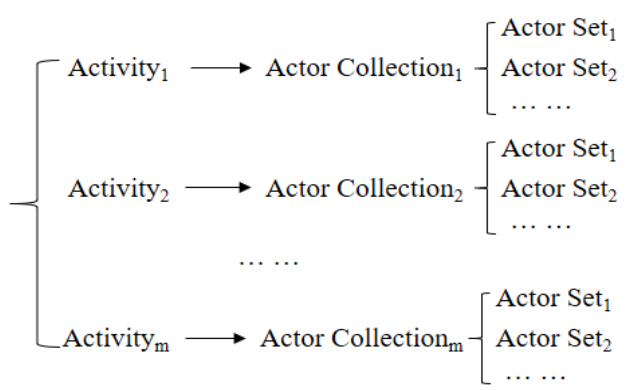

(a)
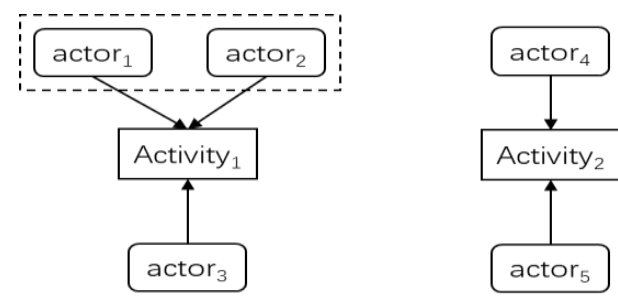

(b)

Figure 2. Example of activity-actor map

For the same activity, multiple actors may be able to perform it. If there are multiple participants in an activity, their relationship may be cooperative or competitive. That is, the activity is completed by the cooperation of several actors, each participant is indispensable to complete the activities, then the actors belong to the cooperative relationship; If the actors are exclusive in completing the activity, the actors compete and complete the activity alone. If different actors execute the activity in different process instances, but do not appear in the same process instance at the same time, it is considered that there is a competitive relationship between the actors. Hereby, participants who complete an activity can be put into a collection, and each element in the collection represents a set of participants competing to execute the activity, which may contain only one participant or multiple participants. Combining the activity information of causal matrix with the actor information extracted from log, we can establish the mapping relationship between activity and actor, as shown in Figure 2 (a). An example of the activity-actor map is shown in Figure 2(b), the participants of activity are $_{\text {actor }}$, actor $\left._{2}\right\}$ or \{actor $_{3\}}$, that is to say, the activity is either completed by the cooperation of Actor 1 and Actor 2 or by Actor 3 alone; Activity 2 is either done by $\left\{a^{2} t_{0}\right\}$ or by $\left\{\right.$ actor $\left._{5}\right\}$. The mapping relationship between activities and actors in Figure 2(b) can be expressed as follow:

$\left\{\right.$ Activity $_{1} \rightarrow\left[\left[\right.\right.$ actor $_{1}$, actor $\left._{2}\right],\left[\right.$ actor $\left.\left._{3}\right]\right]$, Activity $_{2} \rightarrow\left[\left[\right.\right.$ actor $\left._{4}\right],\left[\right.$ actor $\left.\left.\left._{5}\right]\right]\right\}$

In this way, the relationship between activities and actors, as well as between actors and actors, can be clarified.

\section{3) Actor-Value Map}

In the service process, participants generate value added by completing the activities. Value creation is not only related to activities, but also closely related to participants. The same activities, completed by different participants, may produce different value-added. For example, in home appliance repair service, for the same repair work, due to the different skill levels of senior repairmen and ordinary repairmen, not only the time consumption and resource consumption will be different, but also the completion effect will be different, which will affect the value of the activity.

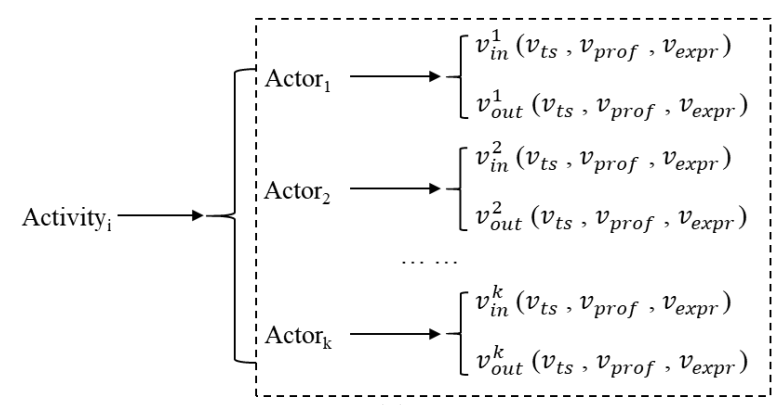

(a)

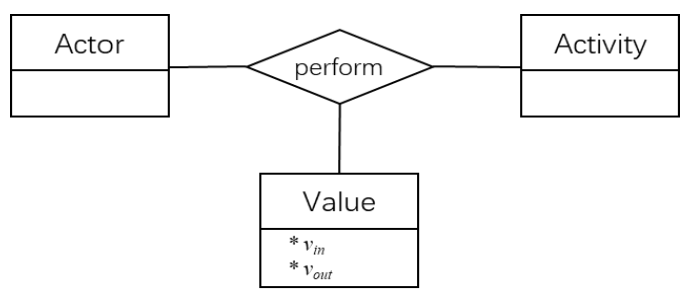

(b)

Figure 3. Actor-value map with activity

The process of an actor performing an activity can be recorded by one or more events. From the attributes of events, we can extract the corresponding general value, domain related value and actor related value. In practice, these attributes may only contain part of the value information, but not all the value information. Then, according to the characteristics of value, the obtained value information is mapped to the three dimensions of service value: time and 
space, profit, and experience []. This is the value generated when actors participate in activities, including input value and output value. Based on this, we can construct the mapping relationship between actors and service value, as shown in the dotted box in Figure 3 (a). Each actor participating in a specific activity has input value $v_{\text {in }}$ and output value $v_{\text {out }}$, which are represented by a triplet of three dimensions of value information, in which $v_{t s}$ represents time and space, $v_{\text {pro }}$ represents interest, and $v_{\text {expr }}$ represents experience. If an actor does not generate value when completing an activity, it can be removed from the collection of actors in the activity.

\section{Specific steps of the algorithm}

According to the idea above, the steps of the serviceoriented improved Heuristics Miner are shown below.

Input: Event log in XES format

Output: Service process value model presented as augmented $\mathrm{C}$-net format

Step 1: Pre-processing of data. Noise and traces that describes incomplete service process will be removed from the event logs, and get a valid event $\log \log { }_{0}$;

Step 2: Parsing the $\log _{0}$ to mine the causality matrix $M_{c}$;

Step 3: Identifying relationships between activities, and building dependency graph $D G$ according to $M_{a}$;

Step 4: Identifying selection, parallelism, and longdistance dependencies in $D G$ to obtain the service process model $S P M$;

Step 5: Extracting the actors and values information from the $\log _{0}$, get the actor set $\{a\}$ and value set $\{v\}$;

Step 6: Constructing activity-actor map $M_{a p}$ based on $M_{a}$ and actor set $\{a\}$;

Step 7: Constructing actor-value map Mapav based on Mapa and actor set $\{v\}$;

Step 8: Annotating the $M a p_{a v}$ into SPM to get the service process value model $S P V M$.

\section{CASE STUDY}

In this section, we use the actual event log of a famous household appliance manufacturer's air conditioning aftersales service process to verify the improved heuristic miner. We use the ProM framework as the experimental environment. The version of ProM framework is 6.9, which was released in 2019 , and its revision is 41850 . The improved heuristic miner is implemented as a plug-in of ProM.

\section{A. The Air Conditioner Repair Service Example}

The service process starts from the customer's application for repair, in which the application for repair is manually applied by the customer through the call center, WeChat applet, mobile service app, or web service market. After receiving the application, the intelligent interaction system will identify the customers according to the customer information, and feedback automatically through the chat robot. If the robot cannot solve the customer's problem correctly, it will be transferred to the manual customer service staff, who will further try to solve the customer's problem. Some simple common problems can be solved at this stage, such as the problem that the air conditioner cannot be turned on because the remote control has no power. For the problem that cannot be solved by the service staff, he or she will issue a service order through the service order center. The service store or maintenance engineer obtains the order through the service order center, and then the repair personnel go to the customer's place for repair at the appointed time. When the repair is completed, it needs to test whether the fault has been fixed. If the fault has not been eliminated, it needs to be repaired again. If the fault is eliminated, the repair service is delivered successfully. It is usually impossible to repair the air conditioner for an unlimited number of times. When the air conditioner cannot be fixed in 3 times, it will be given up and provide customer with replacement service. After the completion of the service, customers can evaluate the service, and finally the system needs to archive the information of the whole repair service. Since the data in the log involves the business operation information of the enterprise and cannot be completely disclosed, we have processed the data. Here is an instance of "Test Repair" activity after processing, as shown in Figure 4.

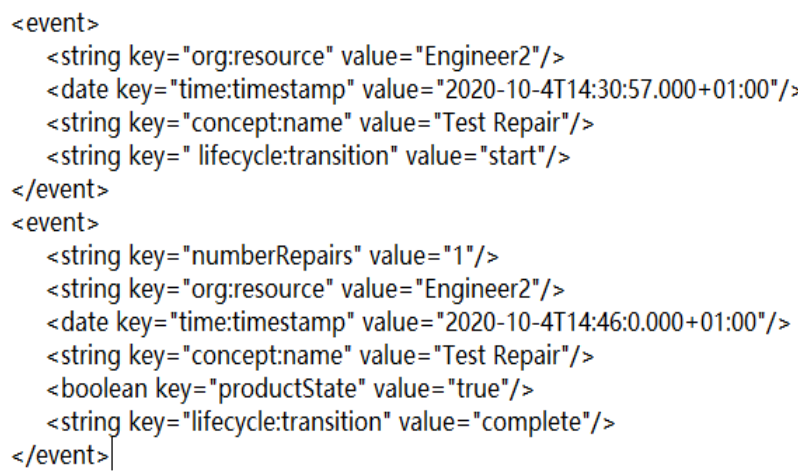

Figure 4. An instance of "Test Repair"

\section{B. Result and evaluation of experiment}

According to the algorithm ideas and steps in the third section, we first deal with the log to remove the nonconforming trace. The process of mining causal matrix and dependency graph is consistent with the original HM algorithm, and we will not repeat it here. In the process of mining causal matrix, we can get all the activities in the service process, and each activity has a corresponding relationship with <event $>$ in the log. Then, the actors of activities are obtained from each <event $>$ attributes with the key "org: resource" and/or "org: role", so that the actor set of all activities can be obtained, and the mapping is constructed based on the relationship between activities and actors. Figure 5 shows part of the activity-actor map of the example. On this basis, we get the value information from the <event $>$ attribute of the actor. For example, we can obtain the time from the attribute with the key of "time: timestamp", the product status information from the attribute with the key of "productstate", and the user's evaluation or experience information from the attribute with the key of "expr: evaluation", etc. Figure 6 shows part of the actor-value map of the example. Finally, the content of actor-value map is annotated into the corresponding activities in the service process, and the construction of service process value model 
can be completed. The service process value model obtained in the example is shown in Figure 7.

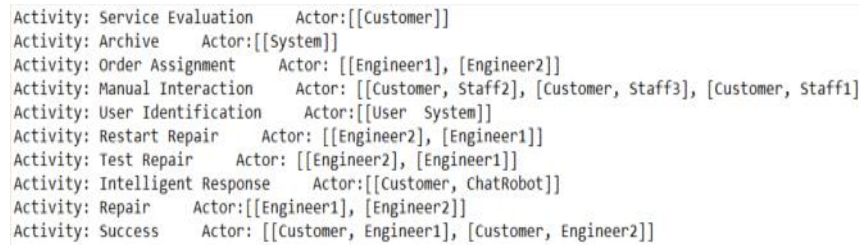

Figure 5. Part of the activity-actor map of the example

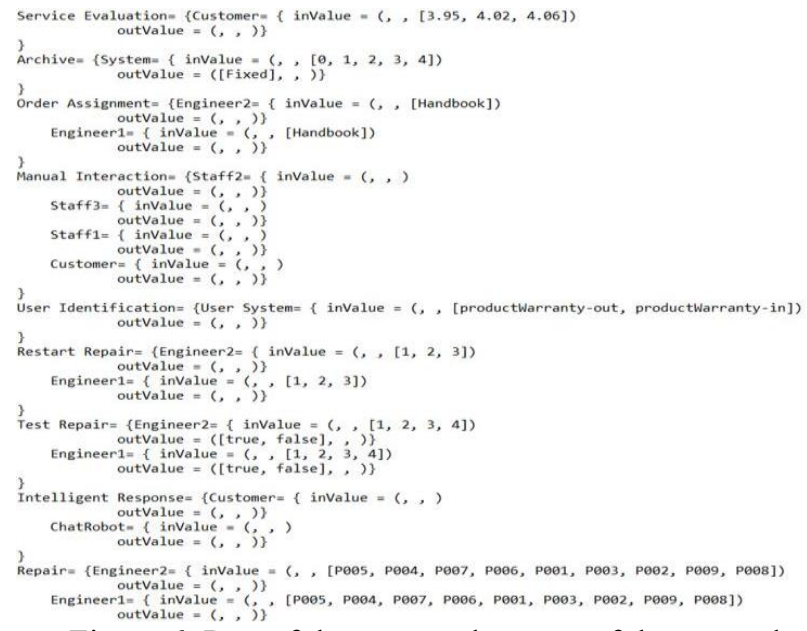

Figure 6. Part of the actor-value map of the example

\section{CONCLUSION}

In this paper, according to the characteristics of service process in product service system and the value creation of service participants in service process, we propose an improved heuristic miner. Through this improved mining algorithm, we can not only mine the service process from the service event log, but also extract the service actors and service value, and build the service process value model. The service process value model shows the relationship between the participants of each activity in the service process and the value created by each participant when completing the activity. It can help enterprises understand the value creation mechanism in the service process and optimize the service.

Although our improved heuristic miner can be applied to the actual service scenarios of manufacturing enterprises, there is still a lot of work to be improved. Because the service process is different from the business process, and the service value involves many attributes in the event log, and there are some interference attributes, we need to continue to study the standardization of service event $\log$ in the future. In addition, the performance of the algorithm and the readability of the service process value model can be further optimized.

\section{ACKNOWLEDGMENT}

This work was supported in part by the National Key Research and Development Program of China (No. 2018YFB1702603), in part by the National Natural Science Foundation of China (No. 61902090, 61772159), and in part by the Natural Science Foundation of Shandong Province under Grant ZR2020MF032.

\section{REFERENCES}

[1] P. P. Wang, X. G. Ming, and M. K. Zheng. "A framework of value creation for industrial product-service." IFIP International Conference on Product Lifecycle Management. Springer, Cham, 2015.

[2] F. H. Beuren, M. G. G. Ferreira, P. A. C. Miguel. "Product-service systems: a literature review on integrated products and services," Journal of cleaner production, vol.47, 2013, pp.222-231.

[3] W.M. Van der Aalst. "Process Mining: Discovery, Conformance and Enhancement of Business Processes," Springer: Berlin, Germany, 2011.

[4] S. Vandermerwe, J. Rada. "Servitization of business: adding value by adding services," European management journal, vol. 6(4), 1988, pp.314-324

[5] M.J. Goedkoop Msc, C. J.G. van Halen MSc, . H. R.M. te Riele MSc and P.J.M. Rommens Msc. "Product Service systems, Ecological and Economic Basics," 1999.

[6] E. Manzini, C. Vezzoli."A strategic design approach to develop sustainable product service systems: examples taken from the 'environmentally friendly innovation'Italian prize," Journal of cleaner production, vol.11(8), 2003, pp. 851-857.

[7] M. T. N. Wong. "Implementation of innovative product service systems in the consumer goods industry," Diss. University of Cambridge, 2004.

[8] M. A. K.Siddike, K. Hidaka. "Toward a Value Metrics-A ServiceDominant Logic View," Advances in The Human Side of Service Engineering. Springer, Cham, 2017, pp.187-197.

[9] Q.T. Zeng. "A survey of research issues and approaches on process mining," Journal of System Simulation, vol.19(16), 2007, pp. 275-280.

[10] A. Weijters, W. M. P. van Der Aalst, A. K. A.De Medeiros. "Process mining with the heuristics miner-algorithm," Technische Universiteit Eindhoven, Tech. Rep. WP 166, 2006, pp.1-34.

[11] A. Weijters, J. T. S. Ribeiro."Flexible heuristics miner (FHM)," 2011 IEEE symposium on computational intelligence and data mining (CIDM). IEEE, 2011, pp.310-317.

[12] X. Zhou, G. Zacharewicz, D. Chen, D. Chu."A Method for Building Service Process Value Model Based on Process Mining," Applied Sciences, vol.10,2020, 7311 .

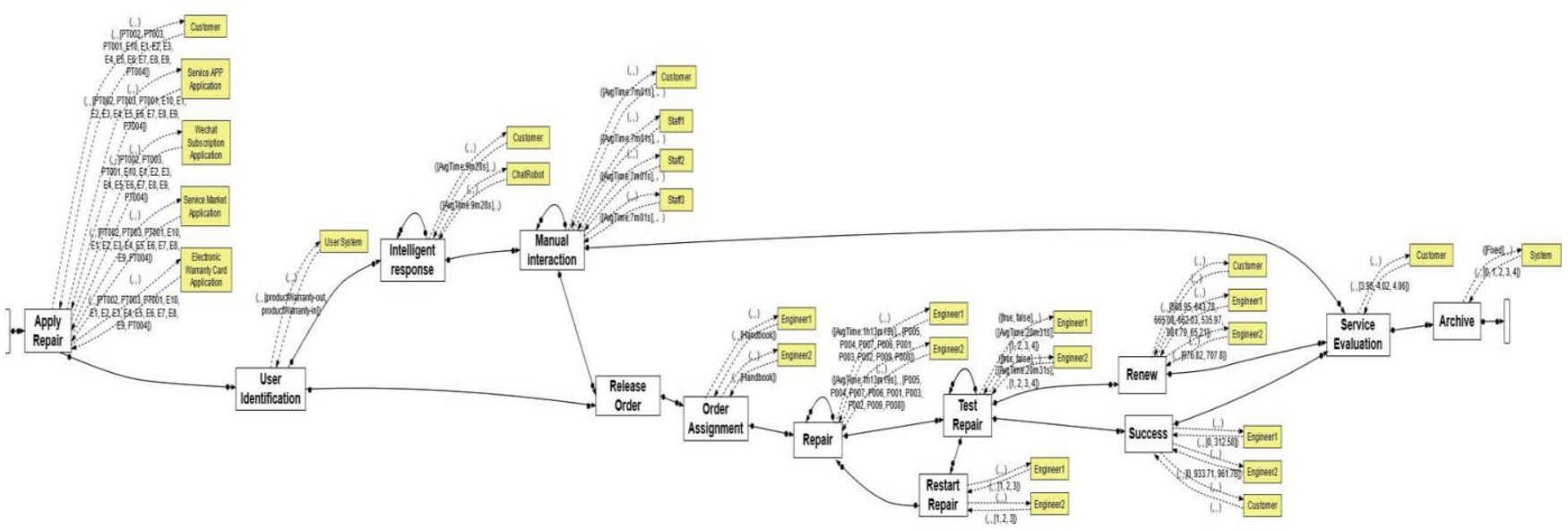

Figure 7. Service process value model of the example 